(C) 2014 IEEE. Personal use of this material is permitted. Permission from IEEE must be obtained for all other uses, in any current or future media, including reprinting/republishing this material for advertising or promotional purposes, creating new collective works, for resale or redistribution to servers or lists, or reuse of any copyrighted component of this work in other works." 


\title{
Bearing Angle Based Cooperative Source Localization
}

\author{
Che $\operatorname{Lin}^{1}$, Guofei Chai ${ }^{1}$, Zhiyun Lin ${ }^{1, *}$, Gangfeng Yan $^{1}$, Guoqiang Mao ${ }^{2}$
}

\begin{abstract}
This paper deals with the cooperative source localization problem with the goal of having an accurate estimate of the coordinate of the source cooperatively by a group of unicycle-type mobile agents. Neither absolute positioning information nor a common sense of direction is shared by the agents. Each agent gets its estimate about the source's coordinate in its own local frame based on the bearing measurements about its neighbors (that may or may not include the source) together with its own linear and angular speed information. A continuous time estimation scheme and a distributed fusion scheme are proposed for this goal such that the source's relative coordinate can be estimated at any time by each agent no matter whether it can directly detect the source or not. The globally asymptotic convergence of the estimation scheme and the fusion scheme is rigorously analyzed. Simulation results are also provided to verify the effectiveness of the proposed algorithms.
\end{abstract}

\section{INTRODUCTION}

Source localization or cooperative source localization has attracted a lot of attention in recent years. It has a number of important applications, for example, formation control, navigation, search and rescue etc. In the formation control problem [1], the followers may need to localize the positions of the leaders in the network, based on which a distributed control law can then be developed to achieve certain desired formation shape. For the navigation problem, every agent may have to localize its own position relative to a landmark in real time so that it can travel from a starting location to a target location along a specific path.

Roughly, cooperative source localization can be classified into two categories, based on whether the agents are stationary or mobile. In the first category, a network of agents remains stationary after they are deployed, usually in sensor networks. For this class, it generally requires three or more nodes to realize cooperative localization about one or many static nodes depending on different types of measurements [2] and about a moving target (such as a jammer in a wireless network) [3], [4]. The second category concerns about a network of mobile agents, for which either the mobile agents use one or multiple source nodes as references to localize themselves, or the mobile agents try to localize one or more targets with the help of their motion information. For this class, mobile agents are capable of localizing themselves with relative measurement of just one source node, and a

The work was supported by Zhejiang Provincial Natural Science Foundation of China LR13F030002 and the Distinguished Visiting Scholars Scheme of The University of Technology Sydney.

${ }^{1}$ State Key Laboratory of Industrial Control Technology, College of Electrical Engineering Zhejiang University, 38 Zheda Road, Hangzhou, 310027 P. R. China.

${ }^{2}$ School of Computing and Communications, The University of Technology, Sydney, Australia.

*Corresponding author: Email: linz@zju.edu.cn single mobile agent is also able to localize a target of interest. Even so, a group of agents working together for cooperative localization is still attractive as such a scenario can improve the localization performance for the case with measurement failures and measurement noises, which are usually unavoidable. Among different kinds of local measurements, bearing measurements have been widely used for localization [5]-[10]. More recently, [8] studies the optimal target localization problem for a sensor network with bearing-only measurements in a constrained three-dimensional underwater scenario. In [5] and [6], a single mobile agent in the plane uses a continuous time localization algorithm to estimate the position of a target based on bearing measurements and achieves circumnavigation around the target. In [10], two mobile agents undergo a planar circular motion to localize each other using bearing measurements with bounded noises.

This paper aims to develop a deterministic estimation scheme for source localization based on bearing measurements as well. But the setup is different. In contrast to [5], [11] and [6], we assume in this paper that no absolute positions of the mobile agents are available, no compass is carried, and the mobile agents are of unicycle model, which are quite common and meet the practical needs. Moreover, it is also common that not all the agents can detect the source directly at any time. However, the goal is to get an accurate estimate for each agent about the source in its own local frame at any time. Towards this objective, firstly, a continuous-time estimation scheme is proposed using the bearing measurement and linear/angular speed information when an agent is able to measure the bearing angle of the source. The continuous-time estimation scheme requires to know the changing rate of the measured bearing angle, for which a linear time-varying differentiator is considered and the input-to-state stability theory is recalled to show the convergence of the estimation scheme together with the time-varying differentiator. Secondly, a consensus-like fusion scheme is designed for every agent to fuse the estimates from its neighbors as well as its own estimate if exists. By doing so, the agents, which are not able to directly measure the bearing angle about the source, can also estimate the source's coordinate in its local frame. As shown in this paper, the estimate resulting from the fusion scheme is asymptotically convergent to the true relative coordinate of the source in the local frame of each agent no matter whether it can or cannot measure the bearing angle about the source.

\section{PROBLEM FORMULATION}

This section introduces several preliminary results and then formulates the cooperative localization problem. 


\section{A. Preliminaries}

Let $\mathcal{G}=(\mathcal{V}, \mathcal{E})$ be an undirected graph of order $N$ with the set of nodes $\mathcal{V}=\{1,2, \ldots, N\}$, a set of edges $\mathcal{E} \subseteq \mathcal{V} \times \mathcal{V}$. We denote by $\mathcal{N}_{i}$ the neighbor set of node $i$. Let $A=\left[a_{i j}\right] \in$ $\mathbb{R}^{N \times N}$ be the adjacency matrix associated with $\mathcal{G}$, for which $a_{i j}=a_{j i}=1$ if $(i, j)$ is an edge in $\mathcal{G}$ and $a_{i j}=0$ otherwise. A diagonal matrix $D=\operatorname{diag}\left\{d_{1}, d_{2}, \ldots, d_{N}\right\} \in \mathbb{R}^{N \times N}$ is called the degree matrix of $\mathcal{G}$, whose diagonal elements $d_{i}=$ $\sum_{j \in \mathcal{N}_{i}} a_{i j}$ for $i=1, \ldots, N$. The Laplacian of the graph $\mathcal{G}$ is defined as $L=D-A$.

To study the cooperative source localization problem, we use an undirected graph $\mathcal{G}$ to describe the sensing relationship of $N$ agents in a group. That is, $(i, j)$ is an edge of $\mathcal{G}$ if and only if agents $i$ and $j$ can mutually measure the bearing angle information about the other and can communicate each other. Let $L$ be the Laplacian of $G$. Moreover, by taking into account the source, we introduce another graph $\overline{\mathcal{G}}$ associated with the system consisting of $N$ mobile agents and the source (labeled 0), which contains $\mathcal{G}$ as its subgraph. We denote by $\overline{\mathcal{N}}_{i}$ the set of agent $i$ 's neighbors in $\overline{\mathcal{G}}$ possibly including node 0 . Node 0 is in $\overline{\mathcal{N}}_{i}$ if and only if agent $i$ has the bearing measurement about the source. Similarly, we define a diagonal matrix $B \in \mathbb{R}^{N \times N}$ to be a source adjacency matrix associated with $\overline{\mathcal{G}}$ with diagonal elements $b_{i}, i \in 1, \ldots, N$, where $b_{i}>0$ if node 0 is a neighbor of node $i$, that is, agent $i$ has the direct bearing measurement about the source and $b_{i}=0$ otherwise. We say $\overline{\mathcal{G}}$ is connected if there exists a path from node 0 to every other node in $\overline{\mathcal{G}}$. Next we recall a known result about the matrix $H=L+B$.

Lemma 2.1 ([12]): The matrix $H=L+B$ is positive definite if $\overline{\mathcal{G}}$ is connected.

Here we give a simple example consisting of 4 agents and one source. The sensing graph $\overline{\mathcal{G}}$ of the system is shown in Fig. 1. we can check that $\overline{\mathcal{G}}$ is connected though $\mathcal{G}$ is not.
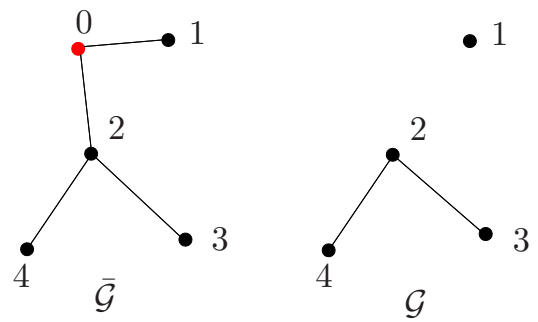

Fig. 1. An example of $\overline{\mathcal{G}}$ and $\mathcal{G}$.

\section{B. Problem Statement}

We consider $N$ mobile agents and suppose that each agent $i$ is of unicycle model and is able to measure its own velocity $v_{i}$ and $w_{i}$ as well as the bearing measurements of its neighbors. Without loss of generality, suppose there is a global coordinate frame $\Sigma_{g}$ and there are local frames $\Sigma_{i}$ 's attached to the agents (see for example, Fig. 2). We use $p_{i}^{g}=\left[\begin{array}{ll}x_{i}^{g} & y_{i}^{g}\end{array}\right]^{\mathrm{T}}$ to represent the coordinate of agent $i$ in the global frame $\Sigma_{g}$. For each agent $i$, the relative coordinate of the source 0 or agent $j$ in agent $i$ 's moving frame $\Sigma_{i}$ is denoted by $p_{i j}=\left[\begin{array}{ll}x_{i j} & y_{i j}\end{array}\right]^{\mathrm{T}}, j=0,1, \ldots, N$. Moreover, we denote $p_{i j}^{g}=p_{j}^{g}-p_{i}^{g}$. Let $\theta_{i} \in \mathbb{R}$ denote the orientation difference between $\Sigma_{i}$ and $\Sigma_{g}$, and let $v_{i}$ and $w_{i}$ denote the linear and angular speed of agent $i$, respectively. The distance $d_{i j}$ between agent $i$ and agent $j$ certainly satisfies $d_{i j}(t)=\left\|p_{i j}(t)\right\|$, where $\|\cdot\|$ refers to the Euclidean norm. An illustration is given in Fig. 2.

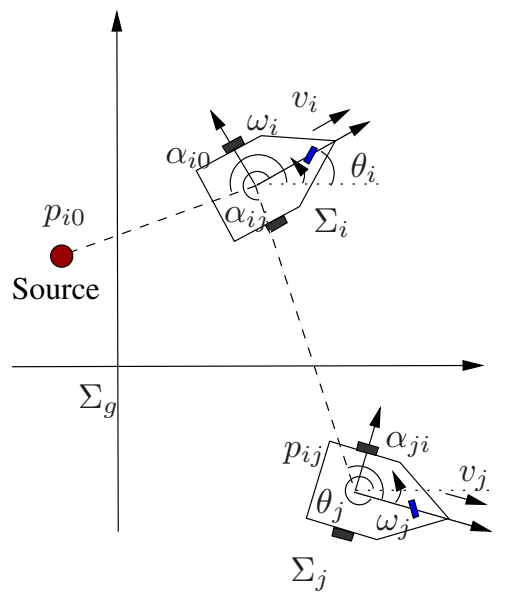

Fig. 2. Local frames and relative states.

In this paper, we assume that

Assumption 2.1: The linear and angular speeds $v_{i}(t)$ and $\omega_{i}(t)$ of each agent $i, i=1, \ldots, N$, are continuously differentiable and bounded.

Finally, we state the cooperative source localization problem using locally available information.

Problem 2.1: Suppose every agent $i \in 1, \ldots, N$ measures the bearing angle $\alpha_{i j}(t) \in[0,2 \pi), j \in \mathcal{N}_{i}$ of its neighbors, its own linear speed $v_{i}(t)$ and angular speed $\omega_{i}(t)$. Besides, each agent receives certain information from its neighbors via communications, (including $v_{j}(t)$ of its neighbor $j$, the bearing angle $\alpha_{j i}(t)$ in its neighbor's local frame, its neighbor's estimate about the source, etc). Design an estimation scheme for every agent $i$ based on these available information to localize the source (namely, $p_{i 0}(t)$ ) in the local frame $\Sigma_{i}$ of agent $i$ ).

\section{LOCALIZATION SCHEME}

In this section, we assume that the bearing angle measurement $\alpha_{i 0}(t)$ about the source is available to agent $i$ all the time. We propose an observer to estimate the coordinate of the source in its local frame. Firstly, the group of $n$ unicycles in the global frame $\Sigma_{g}$ is described by

$$
\left\{\begin{array}{l}
\dot{x}_{i}^{g}=v_{i} \cos \theta_{i} \\
\dot{y}_{i}^{g}=v_{i} \sin \theta_{i} \\
\dot{\theta}_{i}=\omega_{i} .
\end{array}\right.
$$

With respect to the local frame $\Sigma_{i}$ attached to agent $i$ with the $x$-axis aligning to its heading, the following is the coordinate transformation,

$$
p_{i 0}=R\left(-\theta_{i}\right) p_{i 0}^{g},
$$


where

$$
R(\theta)=\left[\begin{array}{cc}
\cos \theta & -\sin \theta \\
\sin \theta & \cos \theta
\end{array}\right]
$$

represents the counterclockwise rotation matrix. According to (2) and (1), we deduce the dynamic equation of $p_{i 0}(t)$ as follows.

$$
\dot{p}_{i 0}(t)=\left[\begin{array}{cc}
0 & \omega_{i} \\
-\omega_{i} & 0
\end{array}\right] p_{i 0}(t)+\left[\begin{array}{c}
-v_{i} \\
0
\end{array}\right] .
$$

Considering the polar coordinate system, the dynamics of $d_{i 0}$ and $\alpha_{i 0}$ can be easily obtained as

$$
\left\{\begin{array}{l}
\dot{d}_{i 0}=-v_{i} \cos \alpha_{i 0} \\
\dot{\alpha}_{i 0}=\frac{v_{i} \sin \alpha_{i 0}}{d_{i 0}}-\omega_{i}
\end{array}\right.
$$

Let $u_{i 0}=\left[\begin{array}{ll}-v_{i} & 0\end{array}\right]^{\mathrm{T}}$. Taking the derivative of both sides of $d_{i 0}^{2}=\left\|p_{i 0}\right\|^{2}$ with respect to time and considering (3), one obtains

$$
\frac{\mathrm{d}}{\mathrm{d} t}\left[d_{i 0}^{2}(t)\right]=2 \dot{p}_{i 0}^{\mathrm{T}}(t) p_{i 0}(t),
$$

and

$$
d_{i 0} \dot{d}_{i 0}=u_{i 0}^{\mathrm{T}} p_{i 0}
$$

In order to use the bearing angle information instead of the range information, we use (4) to get

$$
\frac{-v_{i}^{2} \cos \alpha_{i 0} \sin \alpha_{i 0}}{\dot{\alpha}_{i 0}+\omega_{i}}=u_{i 0}^{\mathrm{T}} p_{i 0} .
$$

Combining (5) and (3), we have

$$
\left\{\begin{array}{l}
\dot{p}_{i 0}(t)=A_{i 0}(t) p_{i 0}(t)+u_{i 0}(t) \\
y_{i 0}(t)=u_{i 0}^{\mathrm{T}}(t) p_{i 0}(t)
\end{array}\right.
$$

where

$$
A_{i 0}(t)=\left[\begin{array}{cc}
0 & \omega_{i} \\
-\omega_{i} & 0
\end{array}\right], \quad y_{i 0}(t)=\frac{-v_{i}^{2} \cos \alpha_{i 0} \sin \alpha_{i 0}}{\dot{\alpha}_{i 0}+\omega_{i}} .
$$

Denote by $\hat{p}_{i 0}(t)$ the estimate of $p_{i 0}(t)$ and denote by $\tilde{p}_{i 0}(t)=\hat{p}_{i 0}(t)-p_{i 0}(t)$ the estimation error. We propose a full order estimator as follows

$\dot{\hat{p}}_{i 0}(t)=A_{i 0}(t) \hat{p}_{i 0}(t)+u_{i 0}(t)+u_{i 0}(t)\left[y_{i 0}(t)-u_{i 0}^{\mathrm{T}}(t) \hat{p}_{i 0}(t)\right]$.

Combining (5) and (6), one obtains the dynamics of the estimation error as follows.

$$
\dot{\tilde{p}}_{i 0}(t)=\left[\begin{array}{cc}
-v_{i}^{2} & \omega_{i} \\
-\omega_{i} & 0
\end{array}\right] \tilde{p}_{i 0}(t) .
$$

The limit of $\hat{p}_{i 0}(t)$ gives an estimate for the coordinate of the source when $\tilde{p}_{i 0}(t)$ converges to zero. We then have the following result.

Theorem 3.1: The zero solution of (7) is exponentially stable if there exist $\epsilon_{1}, \epsilon_{2}>0$ and $T>0$ such that for any $t_{0}>0$

$$
\int_{t_{0}}^{t_{0}+T}\left|\omega_{i}(t)\right| d t \geq \epsilon_{1}, \int_{t_{0}}^{t_{0}+T}\left|v_{i}(t)\right| d t \geq \epsilon_{2} .
$$

Proof: First, we choose a quadratic function $V\left(\tilde{p}_{i 0}\right)=$ $\frac{1}{2} \tilde{p}_{i 0}^{T} \tilde{p}_{i 0}$. It is known that $V\left(\tilde{p}_{i 0}(t)\right)>0$ for $\tilde{p}_{i 0}(t) \neq 0$.
Next, denote $\tilde{p}_{i 0}(t)=\left[\begin{array}{ll}\tilde{x}_{i 0}(t) & \tilde{y}_{i 0}(t)\end{array}\right]^{\mathrm{T}}$. Then we have

$$
\begin{aligned}
\dot{V}\left(\tilde{p}_{i 0}\right) & =\frac{1}{2} \tilde{p}_{i 0}^{\mathrm{T}}\left(\left[\begin{array}{cc}
-v_{i}^{2} & \omega_{i} \\
-\omega_{i} & 0
\end{array}\right]+\left[\begin{array}{cc}
-v_{i}^{2} & -\omega_{i} \\
\omega_{i} & 0
\end{array}\right]\right) \tilde{p}_{i 0} \\
& =\frac{1}{2} \tilde{p}_{i 0}^{\mathrm{T}}\left[\begin{array}{cc}
-2 v_{i}^{2} & 0 \\
0 & 0
\end{array}\right] \tilde{p}_{i 0} \\
& =-v_{i}^{2} \tilde{x}_{i 0}^{2} \leq 0 .
\end{aligned}
$$

$\dot{V}\left(\tilde{p}_{i 0}(t)\right)$ is negative semi-definite and $V\left(\tilde{p}_{i 0}(t)\right)$ is bounded. We check

$$
\begin{aligned}
\ddot{V}\left(\tilde{p}_{i 0}\right) & =-2 v_{i} \dot{v}_{i} \tilde{x}_{i 0}^{2}-2 v_{i}^{2} \tilde{x}_{i 0} \dot{\tilde{x}}_{i 0} \\
& =-2 v_{i} \dot{v}_{i} \tilde{x}_{i 0}^{2}-2 v_{i}^{2} \tilde{x}_{i 0}\left(-v_{i}^{2} \tilde{x}_{i 0}+\omega_{i} \tilde{y}_{i 0}\right) .
\end{aligned}
$$

Because $v_{i}, \dot{v}_{i}, \omega_{i}, \tilde{x}_{i 0}(t)$, and $\tilde{y}_{i 0}(t)$ are all bounded, $\ddot{V}\left(\tilde{p}_{i 0}(t)\right)$ is finite, which means that $\dot{V}\left(\tilde{p}_{i 0}(t)\right)$ is uniformly continuous in time. According to Barbalat's Lemma [13], $\dot{V}\left(\tilde{p}_{i 0}\right)(t) \rightarrow 0$ as $t \rightarrow \infty$. Therefore, $\tilde{x}_{i 0}(t) \rightarrow 0$ as $t \rightarrow \infty$ due to the condition about $v_{i}(t)$ in Theorem 3.1.

Consider $\dot{\tilde{x}}_{i 0}(t)=-v_{i}^{2} \tilde{x}_{i 0}(t)+\omega_{i} \tilde{y}_{i 0}(t)$, and let $f(t)=$ $\tilde{x}_{i 0}(t), \quad g_{1}(t)=\omega_{i} \tilde{y}_{i 0}(t), \quad g_{2}(t)=-v_{i}^{2} \tilde{x}_{i 0}(t)$. Because $\dot{g}_{1}(t)=\dot{\omega}_{i} \tilde{y}_{i 0}(t)-\omega_{i}^{2} \tilde{x}_{i 0}(t)$ is finite (implying that $g_{1}(t)$ is uniformly continuous), and $g_{2}(t) \rightarrow 0$ as $t \rightarrow \infty$, by Extended Barbalat's Lemma [14], it follows that $g_{1}(t) \rightarrow 0$ as $t \rightarrow \infty$. Therefore, using the condition about $\omega_{i}(t)$ in the theorem, we get $\tilde{y}_{i 0}(t) \rightarrow 0$.

In conclusion, we have $\tilde{p}_{i 0}(t) \rightarrow 0$ as $t \rightarrow \infty$, or equivalently, the zero solution of (7) is asymptotically stable. For a linear system, this also means exponential stability [13].

Remark 3.1: Theorem 3.1 shows that during any time interval of certain length, the agent should not keep stationary or move straightly towards the target. Moreover, qualitatively speaking, the larger $\epsilon_{1}$ and $\epsilon_{2}$, the faster $\hat{p}_{i 0}$ converges to $p_{i 0}$.

However, adopting such an approach in practice would require the explicit differentiation of the measurement $\alpha_{i 0}(t)$. In the following, we introduce a linear time-varying (LTV) differentiator to estimate the derivative of $\alpha_{i 0}(t)$. This differentiator does not need any information about the nature of the signal or a prior knowledge of the upper bounds of its higher derivatives.

Lemma 3.1 ( [15] ): Let $\eta(t): \mathbb{R}_{>0} \rightarrow \mathbb{R}$ be a scalar function of class $C^{\infty}$ and let $\left(\rho_{k}, k=0,1,2, \cdots\right)$ be a sequence of positive real numbers. If the higher derivatives of $\eta(t)$ satisfy

$$
\sup _{t \geq 0}\left|\eta^{(k)}(t)\right| \leq \rho_{k}, \quad k=0,1,2, \ldots,
$$

then the state of the following time-varying system

$$
\dot{z}(t)=A(t) z(t)+B(t) \eta(t)
$$

asymptotically converges to $[\eta(t) \dot{\eta}(t)]^{T}$ with

$$
A(t)=\left[\begin{array}{cc}
0 & 1 \\
-a^{2} t^{2} & -2 a t
\end{array}\right], B=\left[\begin{array}{c}
0 \\
a^{2} t^{2}
\end{array}\right], a \in \mathbb{R}_{>0} .
$$

Considering the LTV differentiator, we modify the estimator for each agent as follows, which now does not need to 
know $\dot{\alpha}_{i 0}$.

$$
\left\{\begin{aligned}
\dot{\eta}_{i 0}(t)= & \xi_{i 0}(t) \\
\dot{\xi}_{i 0}(t)= & -a^{2} t^{2} \eta_{i 0}(t)-2 a t \xi_{i 0}(t)+a^{2} t^{2} \alpha_{i 0}(t) \\
\hat{y}_{i 0}(t)= & \frac{-v_{i}^{2} \cos \alpha_{i 0} \sin \alpha_{i 0}}{\xi_{i 0}+\omega_{i}} \\
\dot{\hat{p}}_{i 0}(t)= & A_{i 0}(t) \hat{p}_{i 0}(t)+u_{i 0}(t) \\
& +u_{i 0}(t)\left[\hat{y}_{i 0}(t)-u_{i 0}^{\mathrm{T}}(t) \hat{p}_{i 0}(t)\right]
\end{aligned}\right.
$$

To analyze the convergence of the modified estimator (9), we define $\Delta_{i 0}(t)=u_{i 0}(t)\left(\hat{y}_{i 0}(t)-y_{i 0}(t)\right)$ Then we obtain the following estimation error dynamics.

$$
\dot{\tilde{p}}_{i 0}(t)=\left[\begin{array}{cc}
-v_{i}^{2} & \omega_{i} \\
-\omega_{i} & 0
\end{array}\right] \tilde{p}_{i 0}(t)+\Delta_{i 0}(t) .
$$

According to LTV differentiator we conclude that the disturbance $\lim _{t \rightarrow \infty} \Delta_{i 0}(t)=0$. Then the convergence property of the modified estimator (9) is given below.

Theorem 3.2: If (8) is satisfied, then $\tilde{p}_{i 0}(t)$ in (10) asymptotically converges to 0 .

The proof of Theorem 3.2 uses input-to-state stability theory. Following almost the same argument as the proof of Theorem 2 in [11], Theorem 3.2 can be justified. So the detailed proof is omitted here.

\section{FUSION SCHEME}

In this section, we investigate the cooperative source localization problem using multiple mobile agents.

First we consider how to estimate the coordinate of a neighbor mobile agent in order to use the estimation information about the source passed from the neighbor. The estimation scheme is similar to the one of estimating the source developed in the preceding section, except that the neighbor being localized is not stationary.

Similar with (4), the dynamics of $d_{i j}$ and $\alpha_{i j}$ can be easily obtained as

$$
\left\{\begin{array}{l}
\dot{d}_{i j}=-v_{i} \cos \alpha_{i j}-v_{j} \cos \alpha_{j i} \\
\dot{\alpha}_{i j}=\frac{v_{i} \sin \alpha_{i j}+v_{j} \sin \alpha_{j i}}{d_{i j}}-\omega_{i}
\end{array}\right.
$$

Let $u_{i j}(t)=\left[\begin{array}{ll}v_{j} \cos \theta_{i j}-v_{i} & v_{j} \sin \theta_{i j}\end{array}\right]^{\mathrm{T}}$. Taking the derivative of both sides of $d_{i j}^{2}=\left\|p_{i j}\right\|^{2}$ and following the same procedure for the stationary source, we obtain

$$
\left\{\begin{array}{l}
\dot{p}_{i j}(t)=A_{i j}(t) p_{i j}(t)+u_{i j}(t) \\
y_{i j}(t)=u_{i j}^{\mathrm{T}}(t) p_{i j}(t)
\end{array}\right.
$$

where

$$
\begin{aligned}
& A_{i j}(t)=\left[\begin{array}{cc}
0 & \omega_{i} \\
-\omega_{i} & 0
\end{array}\right] \\
& y_{i j}(t)=-\frac{\left(v_{i} \cos \alpha_{i j}+v_{j} \cos \alpha_{j i}\right)\left(v_{i} \sin \alpha_{i j}+v_{j} \sin \alpha_{j i}\right)}{\dot{\alpha}_{i j}+\omega_{i}} .
\end{aligned}
$$

Here $\theta_{i j}=\theta_{j}-\theta_{i}=\pi+\alpha_{i j}-\alpha_{j i}+2 k \pi$ for some $k \in \mathbb{Z}$.

Thus, similar to (9), we design an estimator for agent $i$ to estimate the relative coordinate of its neighbor $j \in \mathcal{N}_{i}$, using its bearing measurement and the information $\left(\alpha_{j i}\right.$ and $v_{j}$ ) obtained via communication .

$$
\left\{\begin{aligned}
\dot{\eta}_{i j}(t) & =\xi_{i j}(t) \\
\dot{\xi}_{i j}(t) & =-a^{2} t^{2} \eta_{i j}(t)-2 a t \xi_{i j}(t)+a^{2} t^{2} \alpha_{i j}(t) \\
\hat{y}_{i j}(t) & =-\frac{\left(v_{i} \cos \alpha_{i j}+v_{j} \cos \alpha_{j i}\right)\left(v_{i} \sin \alpha_{i j}+v_{j} \sin \alpha_{j i}\right)}{\xi_{i j}(t)+\omega_{i}} \\
\dot{\hat{p}}_{i j}(t) & =A_{i j}(t) \hat{p}_{i j}(t)+u_{i j}(t) \\
& +u_{i j}(t)\left[\hat{y}_{i j}(t)-u_{i j}^{T}(t) \hat{p}_{i j}(t)\right] .
\end{aligned}\right.
$$

Denote by $\tilde{p}_{i j}(t)=\hat{p}_{i j}(t)-p_{i j}(t)$ the estimation error and define $\Delta_{i j}(t)=u_{i j}(t)\left(\hat{y}_{i j}(t)-y_{i j}(t)\right)$. Then we obtain the error system as follows.

$$
\dot{\tilde{p}}_{i j}(t)=-u_{i j} u_{i j}^{\mathrm{T}} \tilde{p}_{i j}(t)+A_{i j}(t) \tilde{p}_{i j}(t)+\Delta_{i j}(t) .
$$

Next we present a sufficient convergence condition for the estimation error system.

Theorem 4.1: The estimate $\hat{p}_{i j}(t)$ in (11) asymptotically converges to $p_{i j}(t)$ if there exist $T$ and $\epsilon_{3}>0$ such that for any $t_{0} \geq 0$,

$\int_{t_{0}}^{t_{0}+T}\left|\omega_{i}(t) u_{i j}^{\mathrm{T}}(t) u_{i j}(t)+\frac{1}{\omega_{i}(t)} u_{i j}^{\mathrm{T}}(t) A_{i j} \dot{u}_{i j}(t)\right| d t \geq \epsilon_{3}$.

Proof: Regarding $\Delta_{i j}(t)$ as the input disturbance, we first analyze the unforced system

$$
\dot{\tilde{p}}_{i j}(t)=-u_{i j}(t) u_{i j}^{\mathrm{T}}(t) \tilde{p}_{i j}(t)+A_{i j}(t) \tilde{p}_{i j}(t) .
$$

We choose a quadratic function $V\left(\tilde{p}_{i j}\right)=\frac{1}{2} \tilde{p}_{i j}^{T} \tilde{p}_{i j}$. It is clear that $V\left(\tilde{p}_{i j}(t)\right)>0$ for $\tilde{p}_{i j}(t) \neq 0$. Taking the derivative of $V\left(\tilde{p}_{i j}\right)$, we have

$$
\begin{aligned}
\dot{V}\left(\tilde{p}_{i j}(t)\right) & =\frac{1}{2} \tilde{p}_{i j}^{\mathrm{T}}(t)\left(-2 u_{i j} u_{i j}^{\mathrm{T}}+A_{i j}^{\mathrm{T}}+A_{i j}\right) \tilde{p}_{i j}(t) \\
& =-\left[u_{i j}^{\mathrm{T}}(t) \tilde{p}_{i j}(t)\right]^{2} .
\end{aligned}
$$

It can be easily verified that $\ddot{V}\left(\tilde{p}_{i j}(t)\right)$ is finite, which means $\dot{V}\left(\tilde{p}_{i j}(t)\right)$ is uniformly continuous. Thus, according to Barbalat's Lemma [13] we conclude that $\lim _{t \rightarrow \infty} \dot{V}(t)=0$ and therefore, $\lim _{t \rightarrow \infty} u_{i j}^{\mathrm{T}}(t) \tilde{p}_{i j}(t)=0$.

Moreover, it can be verified that $\frac{d^{2}}{d t^{2}}\left(u_{i j}^{\mathrm{T}}(t) \tilde{p}_{i j}(t)\right)$ is finite, which means $\frac{d}{d t}\left(u_{i j}^{\mathrm{T}}(t) \tilde{p}_{i j}(t)\right)$ is uniformly continuous. Thus, according to Barbalat's Lemma [13], we have $\lim _{t \rightarrow \infty} \frac{d}{d t}\left(u_{i j}^{\mathrm{T}}(t) \tilde{p}_{i j}(t)\right)=0$.

Denote $u_{i j}=\left[\begin{array}{ll}u_{x} & u_{y}\end{array}\right]^{\mathrm{T}}$ and $\tilde{p}_{i j}=\left[\begin{array}{ll}\tilde{x} & \tilde{y}\end{array}\right]^{\mathrm{T}}$. Then we have

$$
\lim _{t \rightarrow \infty} u_{x}(t) \tilde{x}(t)+u_{y}(t) \tilde{y}(t)=0
$$

$\lim _{t \rightarrow \infty}\left(\dot{u}_{x}(t)-\omega_{i}(t) u_{y}(t)\right) \tilde{x}(t)+\left(\dot{u}_{y}(t)+\omega_{i}(t) u_{x}(t)\right) \tilde{y}(t)=0$.

From the two equations (15) and (16), it can then be obtained that

$$
\begin{aligned}
& \lim _{t \rightarrow \infty}\left(u_{x}(t) \dot{u}_{y}(t)-u_{y}(t) \dot{u}_{x}(t)+\omega_{i}(t)\left\|u_{i j}(t)\right\|^{2}\right) \tilde{x}(t)=0, \\
& \lim _{t \rightarrow \infty}\left(u_{x}(t) \dot{u}_{y}(t)-u_{y}(t) \dot{u}_{x}(t)+\omega_{i}(t)\left\|u_{i j}(t)\right\|^{2}\right) \tilde{y}(t)=0 .
\end{aligned}
$$

From the condition (13), it is equivalent to say that for any time $t_{0}$, we always have $\int_{t_{0}}^{t_{0}+T} \mid u_{x}(t) \dot{u}_{y}(t)-$ $u_{y}(t) \dot{u}_{x}(t)+\omega_{i}(t)\left\|u_{i j}(t)\right\|^{2} \mid d t>\epsilon_{3}$. Then we conclude that $\lim _{t \rightarrow \infty} \tilde{x}(t)=0$ and $\lim _{t \rightarrow \infty} \tilde{y}(t)=0$, i.e., 
$\lim _{t \rightarrow \infty} \tilde{p}_{i j}(t)=0$. Hence the zero solution of (14) is asymptotically stable. Using ISS, we conclude that $\hat{p}_{i j}(t)$ asymptotically converges to $p_{i j}(t)$ because $\Delta_{i j}(t) \rightarrow 0$ as $t \rightarrow \infty$.

Remark 4.1: When the neighbor agent $j$ remains stationary, i.e., $v_{j}=0$, then the estimation error dynamics (12) turns out to be the same form as (10). Also, since $v_{j}=0$, then $u_{i j}=\left[\begin{array}{ll}-v_{i} & 0\end{array}\right]^{\mathrm{T}}$ and the condition (13) degenerates to $\int_{t_{0}}^{t_{0}+T}\left|\omega_{i}(t) v_{i}^{2}(t)\right| d t>\epsilon_{3}$, which can be transformed into the form like (8).

Remark 4.2: For the scenario that agent $i$ and agent $j$ have parallel linear motions with the same constant speed, it is clear that $u_{i j}$ is constant and $\dot{u}_{x}=0, \dot{u}_{y}=0$, and $\omega_{i}=$ 0 . Thus, the condition (13) is not satisfied. Indeed, for this scenario, the system (12) becomes $\dot{\tilde{p}}_{i j}=-u_{i j} u_{i j}^{\mathrm{T}} \tilde{p}_{i j}$ where $u_{i j}$ is a constant vector and thus the the system (12) is not asymptotically stable. This means that the estimator cannot get the accurate estimate for the relative position in this case, which is consistent to the conclusion above inferred from (13).

Next, we develop a distributed fusion scheme for each agent $i$ to estimate the relative coordinate of the stationary source even when the agent may not be able to detect the source. As illustrated in Fig. 3, if agent $i$ is able to estimate

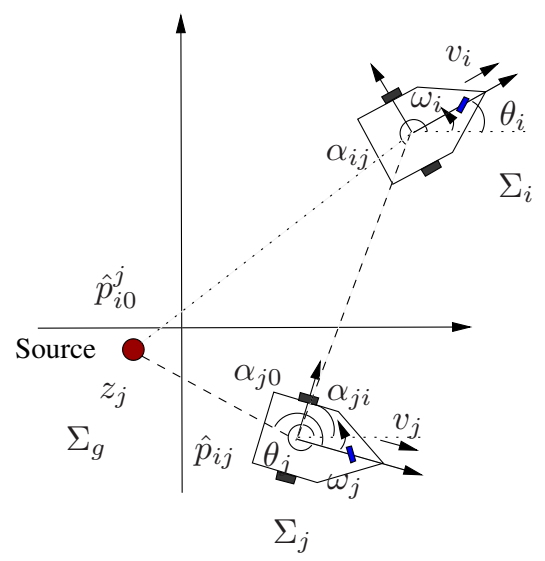

Fig. 3. Indirect estimation of the relative coordinate of the source.

agent $j$ 's relative coordinate and agent $j$ is able to estimate and communicate to agent $i$ the fused estimate for the relative coordinate of the source, denoted as $z_{j}$, in its local frame $\Sigma_{j}$, then the estimate of the source can be indirectly obtained by agent $i$ as

$$
\hat{p}_{i 0}^{j}=\hat{p}_{i j}+R\left(\theta_{i j}\right) z_{j}
$$

where $R\left(\theta_{i j}\right)$ means that agent $j$ needs to rotate the relative coordinate to the local frame $\Sigma_{i}$. Let $b_{i}$ be 1 if agent $i$ has the bearing measurement about the source and 0 otherwise. Then we propose the following consensus-like estimation fusion scheme for agent $i$.

$$
\begin{aligned}
\dot{z}_{i}(t)= & A_{i 0}(t) z_{i}(t)+u_{i 0}(t)+b_{i}\left[\hat{p}_{i 0}(t)-z_{i}(t)\right] \\
& +\sum_{j \in \mathcal{N}_{i}(t)}\left[\hat{p}_{i 0}^{j}(t)-z_{i}(t)\right]
\end{aligned}
$$

where $z_{i}$ represents the fused estimate of the relative coordinate of the source in the local frame $\Sigma_{i}$, and $\hat{p}_{i 0}$ and $\hat{p}_{i 0}^{j}$ are the direct and indirect estimate of the source obtained in (9) and (17), respectively. Then we have the following result.

Theorem 4.2: Suppose the source $\overline{\mathcal{G}}$ is connected. If (8) and (13) are satisfied, then the fused estimate $z_{i}(t)$ in (18) converges to the relative coordinate $p_{i 0}(t)$ of the source.

Proof: For $i=1, \ldots, N$, we let $\tilde{z}_{i}=z_{i}-p_{i 0}, \tilde{p}_{i 0}=$ $\hat{p}_{i 0}-p_{i 0}$, and $\tilde{p}_{i j}=\hat{p}_{i j}-p_{i j}$. Then (18) can be transformed to

$$
\begin{aligned}
\dot{\tilde{z}}_{i}= & \sum_{j \in \mathcal{N}_{i}}\left[R\left(\theta_{i j}\right) \tilde{z}_{j}-\tilde{z}_{i}\right]-b_{i} \tilde{z}_{i} \\
& +A_{i 0}(t) \tilde{z}_{i}+b_{i} \tilde{p}_{i 0}+\sum_{j \in \mathcal{N}_{i}} \tilde{p}_{i j} .
\end{aligned}
$$

This is a typical consensus system, in which $\tilde{z}_{i}$ 's $(i=$ $1, \ldots, N)$ are the individual states of $N$ agents in their own frames. In the following, we transform every $\tilde{z}_{i}$ to a state in a common frame, for example $\Sigma_{g}$. Let $\tilde{z}_{i}^{g}=R\left(\theta_{i}\right) \tilde{z}_{i}$. Then $\tilde{z}_{i}=R\left(-\theta_{i}\right) \tilde{z}_{i}^{g}$. We consider $u_{i}(t)=b_{i} \tilde{p}_{i 0}+\sum_{j \in \mathcal{N}_{i}} \tilde{p}_{i j}$ as an external perturbation. Thus, we obtain

$$
\begin{aligned}
\dot{\tilde{z}}_{i}^{g}= & \dot{R}\left(\theta_{i}\right) \dot{\theta}_{i} \tilde{z}_{i}+R\left(\theta_{i}\right) \dot{\tilde{z}}_{i} \\
= & {\left[\begin{array}{cc}
0 & -\omega_{i} \\
\omega_{i} & 0
\end{array}\right] \tilde{z}_{i}^{g}+\sum_{j \in \mathcal{N}_{i}}\left[\tilde{z}_{j}^{g}-\tilde{z}_{i}^{g}\right]-b_{i} \tilde{z}_{i}^{g} } \\
& +A_{i 0}(t) \tilde{z}_{i}^{g}+R\left(\theta_{i}\right) u_{i}(t) \\
= & \sum_{j \in \mathcal{N}_{i}}\left[\tilde{z}_{j}^{g}-\tilde{z}_{i}^{g}\right]-b_{i} \tilde{z}_{i}^{g}+u_{i}^{g}(t),
\end{aligned}
$$

where $u_{i}^{g}(t)=R\left(\theta_{i}\right) u_{i}(t)$, for which the aggregated system can be expressed as

$$
\dot{\tilde{z}}^{g}=-\left(H \otimes I_{2}\right) \tilde{z}^{g}+u^{g}(t)
$$

where $\tilde{z}^{g}=\left[\tilde{z}_{1}^{g}, \tilde{z}_{2}^{g}, \ldots, \tilde{z}_{N}^{g}\right]^{\mathrm{T}}, u^{g}(t)=\left[u_{1}^{g}, u_{2}^{g}, \ldots, u_{N}^{g}\right]^{\mathrm{T}}$, and $H$ is the matrix as defined in Lemma 2.1. According to Lemma 2.1, $H$ is positive definite, so the zero solution of system (19) is exponentially stable and system (19) is ISS. According to Theorem 4.1 and Theorem 3.1, $u^{g}(t) \rightarrow 0$ as $t \rightarrow \infty$. Then by the input-to-state stability, we have that $\tilde{z}^{g}(t) \rightarrow 0$ as $t \rightarrow \infty$. Finally, we consider $\tilde{z}_{i}=R\left(-\theta_{i}\right) \tilde{z}_{i}^{g}$. Since $R\left(-\theta_{i}\right)$ is non-singular and $\tilde{z}_{i}^{g}(t) \rightarrow 0$ as $t \rightarrow \infty$, we know $\tilde{z}_{i}(t) \rightarrow 0$ as $t \rightarrow \infty$. Thus the conclusion follows.

\section{SIMULATION}

In this section, we present a simulation of four mobile agents for the task of cooperative source localization. To demonstrate the performance and effectiveness of the proposed estimation scheme, we set the stationary source at the origin and let each of the four mobile agents labeled $1,2, \ldots, 4$ take a uniform circular motion around the stationary source labeled 0 along the counterclockwise direction. Both the radius and the moving speeds are 1, 2, 3, and 4 for agent $1,2, \ldots, 4$, respectively. The sensing graph is depicted in Fig. 4. That is, agent 1 and 2 have direct bearing measurements about the source while agent 3 and agent 4 can only have indirect estimate through their neighbors. It 


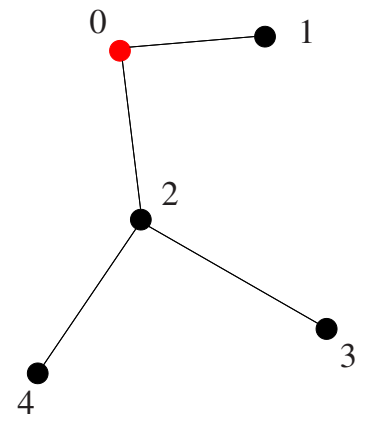

Fig. 4. Sensing graph $\overline{\mathcal{G}}$.

can be simply observed that $\overline{\mathcal{G}}$ is connected. Moreover, it can be verified that for each agent, (8) and (13) hold. We adopt the estimator described in (9), (11) and (18) to estimate the relative coordinate of the source for every agent. Then, by Theorem 4.2, the fused estimate $z_{i}(t)$ converges to the relative coordinate of the source in $\Sigma_{i}$. The evolution curves of the estimation errors $\left\|z_{i}(t)-p_{i 0}\right\|(i=1, \ldots, 4)$ are plotted in Fig. 5, which shows the errors converge to zero.

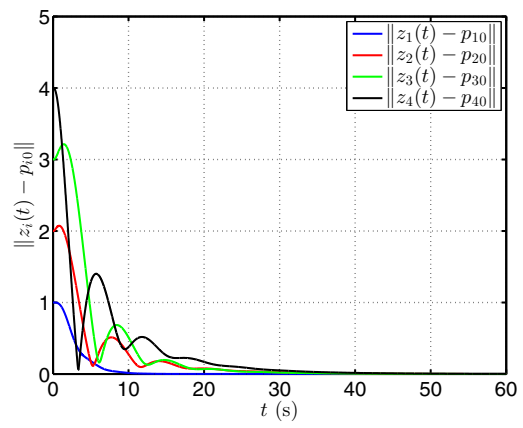

Fig. 5. The evolution of $\left\|z_{i}(t)-p_{i 0}\right\|, i=1,2, \ldots, 4$.

We perform another simulation for our estimation scheme by taking into account the measurement noises. Bounded noises are added to the bearing measurements while the other setup remains the same as for the simulation without noises. The simulation result is shown in Fig. 6, depicting the estimation errors $\left\|z_{i}(t)-p_{i 0}\right\|(i=1, \ldots, 4)$ in the presence of measurement noises. From Fig. 6, we can see that with bounded measurement noises, the estimation errors are also ultimately bounded.

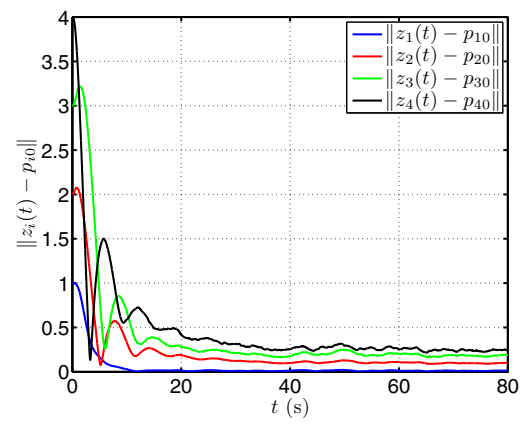

Fig. 6. The evolution of $\left\|z_{i}(t)-p_{i 0}\right\|, i=1,2, \ldots, 4$.

\section{CONCLUSION}

This paper studies the source localization problem based on bearing measurement information. First, an estimator is developed to estimate the relative coordinate of a stationary or a moving object in its local frame with the help of a linear time-varying differentiator. Second, a consensus-like fusion scheme is proposed for cooperative localization by fusing the estimation from the neighboring agents. The proposed estimation scheme only requires the exchange of agents' local information and also their estimates, yet the estimate of every agent is globally asymptotically convergent as long as the sensing graph is connected. There are a few interesting research issues arising from the setup introduced in this paper such as robustness to measurement noises, communication delays, and switching sensing topology.

\section{REFERENCES}

[1] Z. Han, L. Wang, and Z. Lin, "Local formation control strategies with undetermined and determined formation scales for co-leader vehicle networks," in Proceedings of the 52th IEEE Conference on Decision and Control, Florence, Italy, 2013, pp. 7339-7344.

[2] Y. Diao, Z. Lin, and M. Fu, "Localizability and distributed localization of sensor networks using relative position measurements," in Proceedings of the 13th IFAC Symposium on Large Scale Complex Systems: Theory and Applications, Shanghai, China, 2013.

[3] Z. Liu, H. Liu, W. Xu, and Y. Chen, "An error-minimizing framework for localizing jammers in wireless networks," IEEE Transactions on Parallel and Distributed Systems, vol. 25, no. 2, pp. 508-517, 2014.

[4] Z. Zhou, H. Fang, and Y. Hong, "Distributed estimation for moving target based on state-consensus strategy," IEEE Transactions on Automatic Control, vol. 58, no. 8, pp. 2096-2101, 2013.

[5] M. Deghat, I. Shame, B. D. O. Anderson, and C. Yu, "Target localization and circumnavigation using bearing measurements in 2D," in Proceedings of the 49th IEEE Conference on Decision and Control, Atlanta, GA, 2010, pp. 334-339.

[6] M. Deghat, I. Shames, B. D. O. Anderson, and C. Yu, "Localization and circumnavigation of a slowly moving target using bearing measurements," IEEE Transactions on Automatic Control, accepted, 2014.

[7] L. Montesano, J. Gaspar, J. Santos-Victor, and L. Montano, "Cooperative localization by fusing vision-based bearing measurements and motion," in Proceedings of 2005 IEEE/RSJ International Conference on Intelligent Robots and Systems, 2005, pp. 2333-2338.

[8] D. Moreno-Salinas, A. Pascoal, and J. Aranda, "Sensor networks for optimal target localization with bearings-only measurements in constrained three-dimensional scenarios," Sensors, vol. 13, no. 8, pp. 10 386-10 417, 2013.

[9] R. Sharma, S. Quebe, R. W.Beard, and C. N.Taylor, "Bearing-only cooperative localization," Journal of Intelligent \& Robotic Systems, vol. 72, no. 3-4, pp. 429-440, 2013.

[10] M. Ye, B. D. O. Anderson, and C. Yu, "Multiagent self-localization using bearing only measurements," in Proceedings of 2013 IEEE 52nd Annual Conference on Decision and Control, Firenze, Italy, 2013, pp. 2157-2162.

[11] G. Chai, Z. Lin, and M. Fu, "Consensus-based cooperative source localization of multi-agent systems," in Proceedings of the 32nd Chinese Control Conference, Xi'an, China, 2013, pp. 6809-6814.

[12] J. Hu and Y. Hong, "Leader-following coordination of multi-agent systems with coupling time delays," Physica A: Statistical Mechanics and its Applications, vol. 374, no. 2, pp. 853 - 863, 2007.

[13] H. K. Khalil, Nonlinear Systems, 3rd ed. Prentice Hall, 2002.

[14] W. Dixon, D. M. Dawson, E. Zergeroglu, and A. Behal, Nonlinear Control of Wheeled Mobile Robots. Springer, 2001.

[15] S. Ibrir, "Online exact differentiation and notion of asymptotic algebraic observers," IEEE Transactions on Automatic Control, vol. 48, no. 11, pp. 2055-2060, 2003. 